\title{
Perylene-based covalent organic frameworks for acid vapour sensing
}

\author{
Laura Ascherl, ${ }^{1}$ Emrys W. Evans, ${ }^{2}$ Jeffrey Gorman, ${ }^{2}$ Sarah Orsborne, ${ }^{2}$ Derya Bessinger, ${ }^{1}$ Thomas Bein, ${ }^{1}$ Richard $\mathrm{H}$. \\ Friend, ${ }^{2}$ Florian Auras*2 \\ ${ }^{1}$ Department of Chemistry and Center for NanoScience (CeNS), University of Munich (LMU), Butenandtstraße 5-13, 81377 \\ Munich, Germany \\ ${ }^{2}$ Cavendish Laboratory, University of Cambridge, Cambridge CB3 OHE, United Kingdom \\ *e-mail: fa355@cam.ac.uk
}

\begin{abstract}
Traditionally, the properties and functions of covalent organic frameworks (COFs) are defined by their constituting building blocks, while the chemical bonds that connect the individual subunits have not attracted much attention as functional components of the final material. We have developed a new series of dual-pore perylene-based COFs and demonstrate that their imine bonds can be protonated reversibly, causing significant protonation-induced colour shifts towards the nearinfrared, while the structure and crystallinity of the frameworks are fully retained. Thin films of these COFs are highly sensitive colorimetric acid vapour sensors with a detection limit as low as $35 \mu \mathrm{g} \mathrm{L}^{-1}$ and a response range of at least four orders of magnitude. Since the acidochromism in our COFs is a cooperative phenomenon based on electronically coupled imines, the COFs can be used to determine simultaneously the concentration and protonation strength of non-aqueous acid solutions, in which $\mathrm{pH}$ electrodes are not applicable, and to distinguish between different acids. Including the imine bonds as functiondetermining constituents of the framework provides an additional handle for constructing multifunctional COFs and extending the range of their possible applications.
\end{abstract}

\section{INTRODUCTION}

Covalent organic frameworks (COFs) are two-dimensional ${ }^{1-3}$ or three-dimensional ${ }^{4-5}$ crystalline, fully organic networks, in which the key physical properties, such as the type and energy of optical transitions ${ }^{6-7}$ or the electrical conductivity ${ }^{8}$ 10 are highly tuneable. These materials can also be seen as a periodic lattice of extremely well defined pores. ${ }^{11-14}$ The environment that a guest molecule encounters in these pores can vary over a wide range, e.g., it can be hydrophilic or hydrophobic or can include different functional groups on the pore walls. ${ }^{15-17}$

When constructing new COFs and defining their properties, the focus lies traditionally on the choice of appropriate building blocks, such as aryls, ${ }^{18-19}$ (fused) thiophenes, ${ }^{20-22}$ or other extended heteroarenes. ${ }^{23-25}$ The bond that connects them, however, is often considered a necessary evil. The linkage chemistry is selected to be reversible enough to provide the required self-healing capabilities during the COF synthesis, yet yield a sufficiently stable framework in order to avoid fast degradation during the intended application.

Apart from these structural aspects, the bond is primarily meant to interfere as little as possible with the function of the framework. In fact, the linkage chemistry is often regarded as one of the limiting factors for potential applications of COFs, especially in terms of their practicability in optoelectronics or electrochemical applications. Non-conjugated bonds such as boronate esters have little impact on the adjacent building blocks, but impede in-plane electronic communication and charge transport. ${ }^{26-27}$ Conjugated linkages such as the very popular imines, on the other hand, can enable electronic communication across the bond, but act as very strong acceptors, causing extremely fast non-radiative quenching of excited states. ${ }^{18,20,28}$ Recently, strategies to overcome these adverse characteristics of imines via post-synthetic transformations into oxazoles, thiazoles, or quinolines have been developed. ${ }^{29-31}$ However, little effort has been undertaken thus far to make use of the imine bonds themselves and employ them as a functional part of the final framework.

Here we utilize the lone electron pair of the imine linkages as the function-determining moiety of a new series of twodimensional COFs. The COFs are based on a newly developed perylene tetraaniline building block and adopt a star-shaped dual-pore topology. Protonation of the imine bonds in these materials causes significant absorption red-shifts and the formation of protonation-induced absorption bands in the near-infrared, while the structure and crystallinity of the frameworks are fully retained. We find that the acidochromism depends on both acid strength and concentration, allowing us to use this effect for sensing and distinguishing between acids in solution and in the vapour phase. We demonstrate that perylene COF thin films can serve as highly sensitive colorimetric acid vapour sensors with a response range of at least four orders of magnitude. 

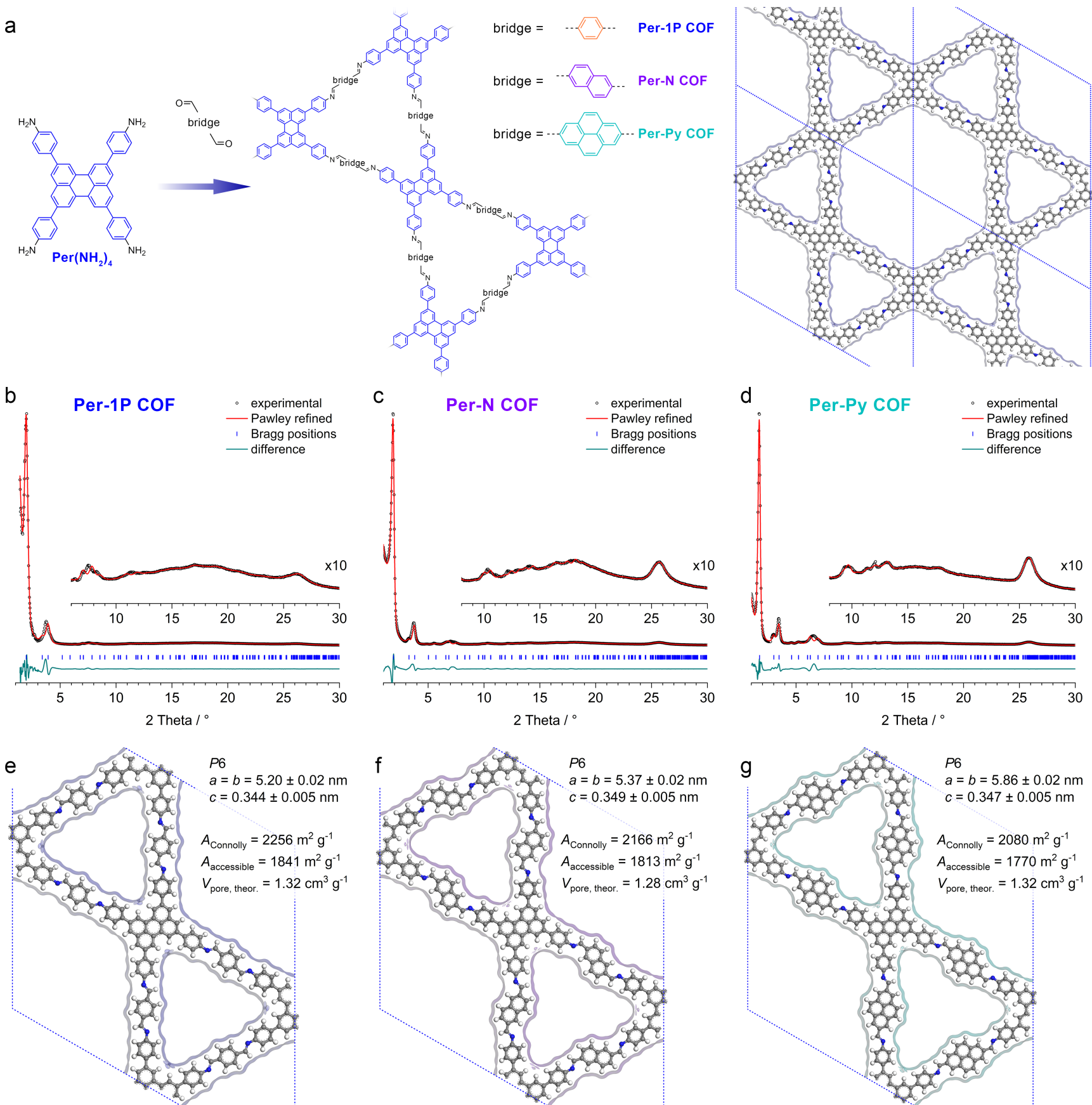

Figure 1. Construction of the new perylene COFs. (a) The solvothermal co-condensation of the perylene tetraaniline with linear dicarbaldehydes yields the star-shaped dual-pore Per-1P, Per-N and Per-Py COFs. (b-d) Experimental PXRD patterns of the perylene COFs (black symbols). Pawley refinements (red lines) provide a good fit with only minimal differences (green) between the experimental and the refined patterns. Bragg positions are indicated by blue ticks. (e-g) Force-field derived, Pawley refined structure models of the three perylene COFs. The Connolly surfaces are calculated for a nitrogen-sized probe $(r=0.184 \mathrm{~nm})$.

\section{RESULTS AND DISCUSSION}

While the range of tetradentate aldehyde building blocks for 2D COFs has expanded significantly over the past years, tetraamines are still limited to only three building blocks, i.e. the pyrene, ethene and porphyrin tetraanilines. ${ }^{12,16,26}$ In order to extend this range towards larger aromatic units, we have developed a perylene-based building block. The 2,5,8,11tetrakis(4-aminophenyl)perylene $\left(\operatorname{Per}\left(\mathrm{NH}_{2}\right)_{4}\right)$ generates starshaped COFs with small trigonal and large hexagonal pores (Figure 1).

The new perylene COFs were synthesized via solvothermal co-condensation of $\operatorname{Per}\left(\mathrm{NH}_{2}\right)_{4}$ with the phenylene-, naphthalene-, and pyrene-based dicarbaldehydes (see the Supporting Information, section B for experimental details). The powder X-ray diffraction (PXRD) patterns of the COFs show sharp reflections and several well-resolved higher-order reflections (Figure $1 b-d$ ). Due to the very large unit cells of these COFs, the overlap between some reflections even at low diffraction angles is unavoidable. The COFs were indexed and Pawley-refined in the hexagonal space group $P 6$ with three symmetry-equivalent perylenes per unit cell (Figure 1eg).

For comparison purposes we also synthesized a range of COFs based on the literature-known tetraphenylpyrene, ${ }^{7,16,18}$ tetraphenylethene, ${ }^{3,12}$ and triphenyltriazine ${ }^{32}$ building blocks. The syntheses and structures of these COFs are shown in the SI, sections $B$ and $D$.

In order to facilitate the spectroscopic analysis and application as sensors, the COFs were also grown as thin films on fused silica, sapphire, or indium-tin-oxide coated glass substrates (see the SI, Section B for experimental details). 2D grazing-incidence wide angle X-ray scattering (GIWAXS) 
measurements confirm that the films consist of the crystalline COFs (SI, Figure S4). For the perylene COFs we observe that the intensities of the $h k 0$ reflections are distributed almost evenly across semicircles with only slightly higher intensities close to the substrate horizon, indicating that these COF films are polycrystalline with random crystallite orientation (SI, Figure S4-1). This is in sharp contrast to the other COFs in this study and other reported COF films, ${ }^{33-34}$ which are highly textured with their respective crystallographic $a-b$ planes parallel to the substrate. We attribute this random orientation to the significantly higher reactivity of the
Per $\left(\mathrm{HN}_{2}\right)_{4}$ building block compared to the pyrene, ethene, or triazine anilines. The increased reactivity causes a rapid growth of the initially formed nuclei on the substrate without the typical equilibrium between imine condensation and dissolution that provides the selectivity for $a-b$ oriented domains. An additional factor could be nuclei that form in the reaction mixture and are subsequently incorporated into the growing COF film. The resulting COF films, however, are optically transparent and homogeneous with only minimal light scattering. a

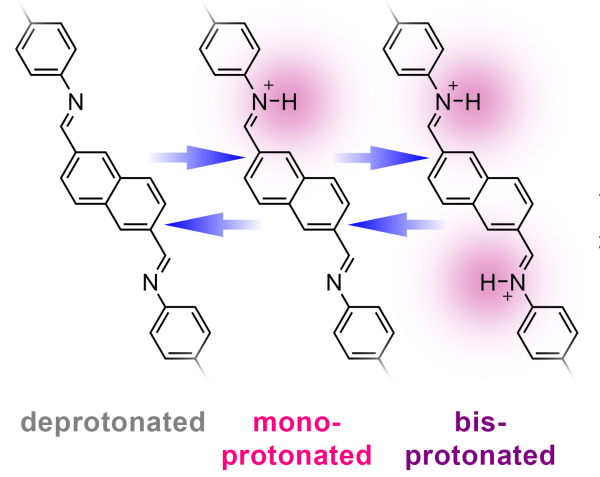

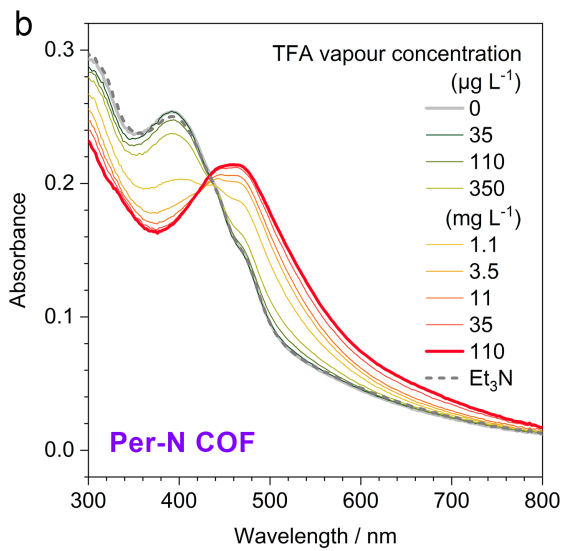

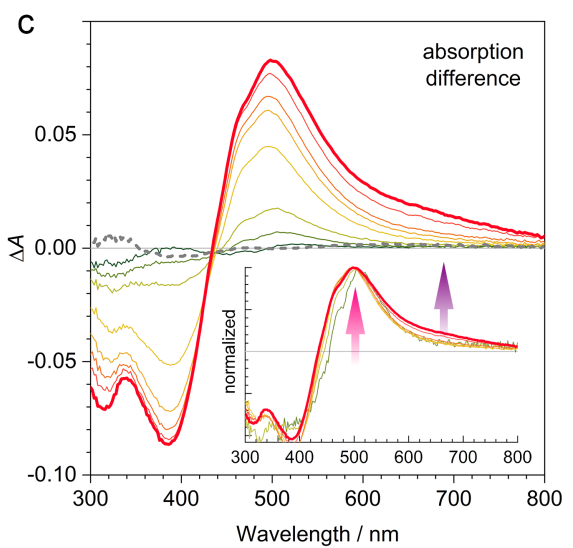

Figure 2. Protonation of the Per-N COF with TFA vapour. (a) The imine-linked bridges of the Per-N COF can be protonated stepwise, causing a fully reversible colour change from yellow to dark brown. (b) Absorption spectra of a Per-N COF film upon protonation in TFA vapour of increasing concentration (green to red lines). The COF film has a response range of at least four orders of magnitude and the protonation can be fully reversed by exposing the protonated COF to triethylamine vapour (grey dashed line). (c) Absorption difference between the protonated and fully deprotonated COF showing a protonation-induced absorption band across the visible and near IR range, accompanied by a bleach band in the blue and UV. Inset: Absorption difference spectra normalized to their respective maximum. The absorption feature formed at low TFA concentrations peaks at $500 \mathrm{~nm}$ with almost no absorption above $600 \mathrm{~nm}$. At higher acid concentrations, however, an additional absorption feature develops in the $600-800 \mathrm{~nm}$ region. We attribute these two features to the mono-protonated and the bisprotonated imine-linked bridges, respectively (see discussion below). Abbreviations: TFA = trifluoroacetic acid.

The nitrogen lone pair of imines can be protonated by sufficiently strong acids, ${ }^{35}$ but this process typically leads to their decomposition if small amounts of water are present. We found, however, that the close-packed structures of 2D COFs provide sufficient stabilisation to render them stable against hydrolysis and enable the fully reversible protonation and deprotonation (Figure 2a). The protonated perylene, pyrene and triazine COFs can be stored in humid air for several hours without any noticeable degradation. Only the 4PE-TT COF, where the steric stabilisation is reduced due to its twisted imine-linked bridges and significantly larger layer spacing ( $4.6 \mathrm{~nm}$ vs. ca. $3.5 \mathrm{~nm}$ for the other COFs) is unstable towards humidity when protonated. A video showing the repeated protonation and deprotonation of a pyrene COF film is supplied with the SI.

The protonation of the imine bonds can be achieved by exposing the COF powders or thin films to acid solutions or vapours and can be followed by infrared spectroscopy (SI, Figure S7). We observe the appearance of the $\mathrm{C}=\mathrm{NH}^{+}$ stretching vibration around $1650 \mathrm{~cm}^{-1}$, accompanied by an attenuation of the imine $\mathrm{C}=\mathrm{N}$ stretching mode around 1610 $\mathrm{cm}^{-1} \cdot{ }^{36}$

The lowest-energy optical transitions of imine-linked COFs have significant charge-transfer character with the ground state electron density being distributed across the frameworks, while the first singlet excited state is more localised on the imines (SI, Figure S14). Since the protonated imines are stronger electron acceptors than their free-base counterparts, the energies of these transitions are lowered, leading to red-shifted absorption and emission spectra. Most COFs are constructed with two electronically coupled imines per bridge unit. In this case the simultaneous protonation of both imines increases the charge transfer character even further and enables an additional stabilisation via quinoidal resonance structures.

For the perylene COF series we observe a colour change from greenish-yellow to dark brown, caused by the appearance of a protonation-induced absorption band in the $450-800 \mathrm{~nm}$ region that is accompanied by a bleach band below $450 \mathrm{~nm}$ (SI, Figure S5-1). Similar colour shifts are observed for the other COFs (SI, Figures S8 and S14a).

In their free-base form the perylene COFs possess a red (Per$1 \mathrm{P}$ and Per-N COFs) or yellow (Per-Py COF) photoluminescence (PL) with comparably long PL lifetimes (SI, Figures S5-2 and S6). If protonated, however, the PL of the Per-Py COF is shifted drastically from $570 \mathrm{~nm}$ to about 1000 $\mathrm{nm}$ and is greatly reduced in intensity. The emission from the other protonated COFs is below the detection limit of our IR detectors.

Building on these initial observations, we investigated the potential of a Per-N COF film as an acid vapour sensor. The COF film was placed in a sealed cuvette and a defined acid atmosphere was generated by evaporating $1 \mu \mathrm{L}$ of a trifluoroacetic acid (TFA) solution in tetrahydrofuran, yielding acid vapour concentrations between $35 \mathrm{\mu g} \mathrm{L}^{-1}$ and $110 \mathrm{mg} \mathrm{L}^{-1}$. Even the lowest acid concentration causes a measurable 
change in the absorption spectrum with protonation-induced absorption and bleach bands extending from 440 to $800 \mathrm{~nm}$ and below $440 \mathrm{~nm}$, respectively (Figure $2 \mathrm{~b}, \mathrm{c}$ ). These bands become more intense with increasing acid vapour concentration, without any saturation effects. We observe, however, the gradual evolution of an additional IR component as the acid vapour concentration is increased (Figure 2c, inset). We attribute these spectral changes to the protonation of only one of the two imines per bridge $(500 \mathrm{~nm}$ absorption) at low TFA concentrations, whereas at higher TFA concentrations also the bis-protonated state $(600-800 \mathrm{~nm}$ absorption) is formed. The stepwise protonation will be discussed in more detail below.

The protonation can be fully reversed by exposing the COF to triethylamine vapour, followed by washing in acetonitrile to remove the triethylammonium salt (Figure 2b,c dashed grey line). To conclude, the Per-N COF acts as a very sensitive colorimetric acid vapour sensor with a low detection limit and a response range of at least four orders of magnitude.
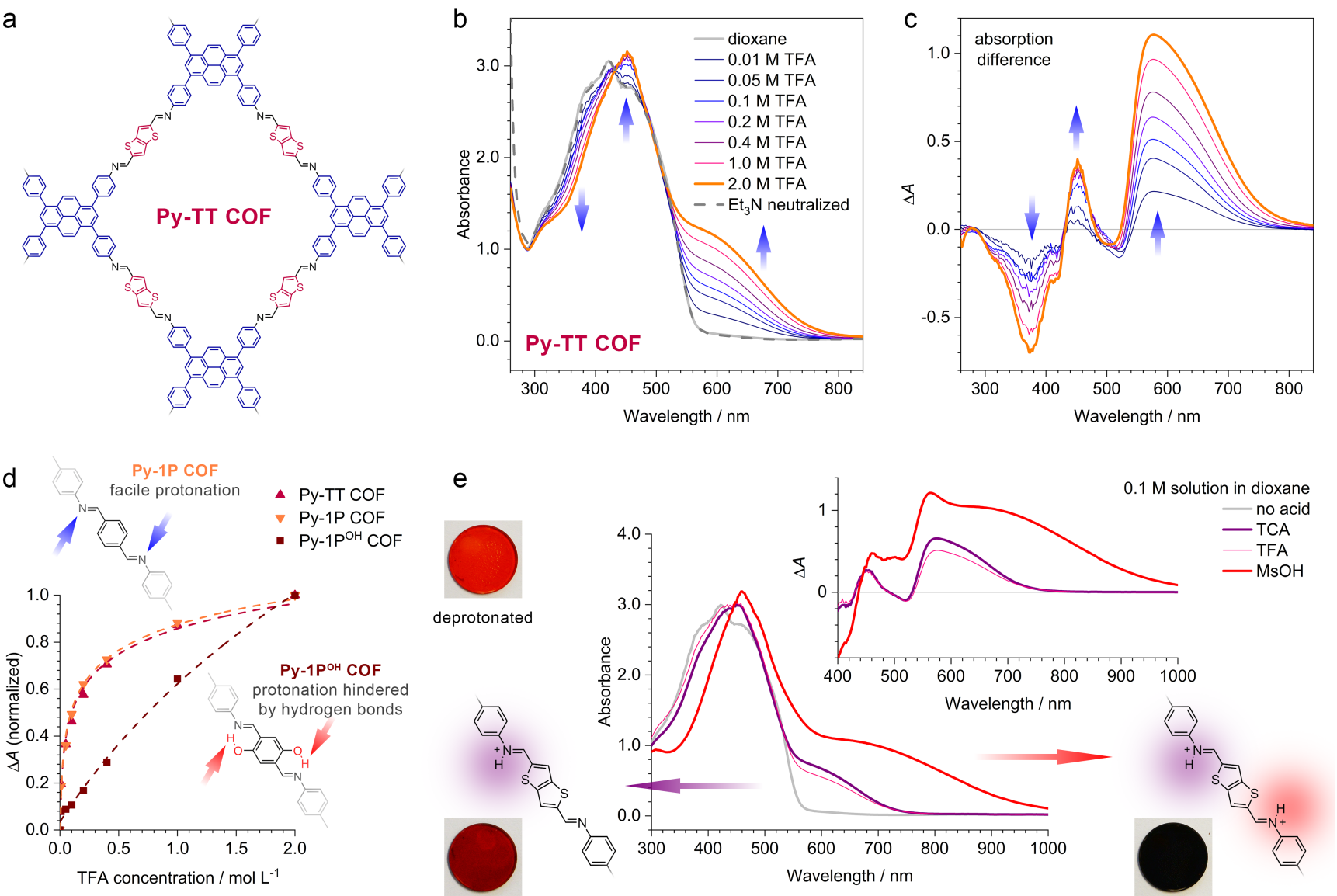

Figure 3. Protonation of pyrene COF films with acid solutions. (a) Chemical structure of the Py-TT COF. The structures of the Py-1P and the $\mathrm{Py}-1 \mathrm{POH}$ COFs are shown in the SI, section B. (b) Absorption changes of the Py-TT COF film upon protonation with TFA solutions of various concentrations in 1,4-dioxane. The protonation can be fully reversed by neutralisation with $\mathrm{Et}_{3} \mathrm{~N}$ (grey dashed line). (c) Plot of the absorption difference between the protonated and the non-protonated Py-TT COF showing the absorption features of the mono-protonated bridge (see discussion below). (d) Evolution of the main absorption bands of the pyrene COFs, determined at their respective maxima (Py-TT COF: 578 nm, Py-1P COF: 544 nm, Py-1POH COF: $590 \mathrm{~nm}$ ). While the absorption change of the Py-TT and Py-1P COFs follows a logarithmic dependence on the acid concentration, the protonation of the $\mathrm{Py}-1 \mathrm{POH}$ COF is considerably retarded due to the $-\mathrm{OH} \cdot \mathrm{N}=$ hydrogen bonds. (e) Protonation of the Py-TT COF with different acid solutions. TFA and TCA solutions produce selectively the mono-protonated bridge unit with a characteristic absorption band between 550 and $750 \mathrm{~nm}$. The considerably stronger $\mathrm{MsOH}$ generates a much stronger absorption band which extends further into the IR, indicating the formation of the bis-protonated species. Insets, photographs of the deprotonated, monoprotonated, and bis-protonated Py-TT COF. Abbreviations: TCA = trichloroacetic acid, TFA = trifluoroacetic acid, $\mathrm{MsOH}=\mathrm{methanesulfonic}$ acid.

For a more detailed analysis of how the COF protonation proceeds we focus on a series of the well-established pyrene COFs (Figure 3a). The Py-TT COF is a donor-acceptor variant of the archetypical Py-1P COF that forms particularly crystalline films. ${ }^{7,18}$ In the $\mathrm{Py}-1 \mathrm{P}^{\mathrm{OH}}$ COF the hydroxy groups provide an additional stabilisation of the structure via hydrogen bonding to the adjacent imine nitrogens. ${ }^{16,37}$ Combining the pyrene tetraaniline with the pyrene tetraaldehyde yields the Py-Py COF with only one imine bond per bridge (SI, Figure S12a). ${ }^{7}$

If the protonation is carried out using TFA solutions in dioxane rather than acid vapour, we observe a gradual colour change of the Py-TT COF from initially orange to dark brown due to the appearance of a strong absorption band between 500 and $750 \mathrm{~nm}$ (Figure $3 \mathrm{~b}, \mathrm{c}$ ). An additional absorption band is formed at $450 \mathrm{~nm}$, accompanied by a bleach band around $370 \mathrm{~nm}$. The intensity of the absorption and bleach bands follows a logarithmic dependence on the acid concentration (Figure 3d, red triangles). The spectral features, however, remain unaffected by the acid concentration, suggesting that under these protonation conditions, only a single, well-defined protonated state is formed. The protonation can be fully reversed by immersing the COF film in a dilute $\mathrm{Et}_{3} \mathrm{~N}$ solution. During the protonation and subsequent deprotonation, the 
crystallinity and porosity of the COFs and COF films are fully retained (SI, Figure S11).

The protonation behaviour of the Py-1P COF is very similar, whereas the protonation of the $\mathrm{Py}-1 \mathrm{P}^{\mathrm{OH}} \mathrm{COF}$ is significantly delayed, highlighting its stabilisation via $-\mathrm{OH} \cdot \cdot \mathrm{N}=$ hydrogen bonds that counteract the imine protonation (Figure $3 \mathrm{~d}$ and SI, Figure S8).

When comparing the absorption spectra obtained upon protonating the COFs with dilute solutions of various acids, however, we notice considerable differences (SI, Figure S10). The weakest acids (acetic acid and chloroacetic acid) do not cause any considerable protonation. The stronger halogenated acetic acids (dichloroacetic acid, trichloroacetic acid, TFA) can mono-protonate the Py-TT and Py-1P COFs (and the $\mathrm{Py}-1 \mathrm{P}^{\mathrm{OH}}$ COF to some degree), producing the spectral features discussed above. Exposing the COFs to these acids at higher concentrations yields the same absorption signatures with higher intensities. The even stronger $p$-toluenesulfonic acid and methanesulfonic acid, however, do not only produce more intense absorption bands than the other acids at the same concentration, but also give rise to significantly different spectral features that extend much further into the IR. The same IR-extended absorption bands can also be obtained with saturated TFA vapour or other strong acids (SI, Figure S13), or TFA solutions in solvents that are very poor hydrogen bond acceptors (SI, Figure S9), ${ }^{38}$ suggesting that their formation depends on the protonation strength of the respective acid mixture rather than the choice of the acid anion.

The observations above strongly suggest that the protonation proceeds in two distinct steps with characteristic absorption bands extending to about $750 \mathrm{~nm}$ and beyond $1000 \mathrm{~nm}$, respectively, in the case of the Py-TT COF. No intermediates or gradual absorption shifts are observed. We attribute these steps to the mono- and the bis-protonation of the two electronically coupled imines within one phenylene- $\mathrm{N}=\mathrm{CH}-$ bridge- $\mathrm{HC}=\mathrm{N}$-phenylene unit (Figure 3e). The first protonation can be achieved with a variety of acid solutions. However, this mono-protonation reduces the basicity of the neighbouring imine and thus considerably more protonation strength is required for the second protonation. This cooperative phenomenon involving electronically coupled imines sets our COFs apart from previous examples of acidochromic framework materials, which rely on isolated acidochromic building blocks. ${ }^{39-40}$

The assignments of the spectra to the mono- and bisprotonated imine bridges are further supported by comparison with the Py-Py COF (SI, Figure S12). Since this framework contains only one imine bond per bridge, we do not expect to observe the second protonation step with stronger acid solutions. Indeed, only one protonated state is formed regardless of the protonation strength of the acid. In addition to the COF films being very sensitive acid vapour sensors, the balance between the mono- and bis-protonated states provides additional information about the respective acid mixture. COF films could be employed as colorimetric probes for determining simultaneously the concentration and

the protonation strength of non-aqueous acid solutions, which are incompatible with standard $\mathrm{pH}$ electrodes. The close-packed crystalline COF lattice provides the stability, molecular-scale porosity and easy read-out required for reliable sensing.

\section{CONCLUSION}

We have developed a series of star-shaped dual-pore perylene-based COFs and have demonstrated that their imine bonds can be reversibly protonated, causing significant colour shifts. Protonation of the phenylene- $\mathrm{N}=\mathrm{CH}-$ bridge $-\mathrm{HC}=\mathrm{N}-$ phenylene subunits proceeds in a two-step process with the second protonation requiring significantly stronger acids. The mono- and the bis-protonated species have distinct absorption features in the red and near-infrared spectral regions, enabling the COFs to act as colorimetric sensors for simultaneously determining the acid strength and concentration in non-aqueous solutions where $\mathrm{pH}$ electrodes are not applicable. Moreover, the COF films are highly sensitive acid vapour sensors with a very low detection limit and a response range of at least four orders of magnitude. By using the imine bond itself as a function-determining moiety, we have provided an additional handle for constructing functional COFs for a broad range of applications.

\section{ASSOCIATED CONTENT}

\section{Supporting Information}

The Supporting Information is available free of charge on the ACS Publications website at DOI: xxxx.

Experimental methods, synthetic procedures, structure analysis and additional spectroscopic data (.pdf). Video of the reversible protonation (.mpg).

\section{AUTHOR INFORMATION \\ Corresponding Author \\ *fa355@cam.ac.uk \\ ORCID}

Emrys W. Evans: 0000-0002-9092-3938

Jeffrey Gorman: 0000-0002-6888-7838

Thomas Bein: 0000-0001-7248-5906

Richard H. Friend: 0000-0001-6565-6308

Florian Auras: 0000-0003-1709-4384

\section{Notes}

The authors declare no competing financial interest.

\section{ACKNOWLEDGMENTS}

This project has received funding from the European Research Council (ERC) under the European Union's Horizon 2020 research and innovation programme (grant agreement No 670405). The research leading to these results has received funding from the European Research Council under the European Union's Seventh Framework Programme (FP7/2007-2013/ERC grant agreement No 321339). We thank Prof. Matthias Thommes for fruitful discussions.

\section{REFERENCES}

1. Côte, A. P.; El-Kaderi, H. M.; Furukawa, H.; Hunt, J. R.; Yaghi, O. M., Reticular Synthesis of Microporous and Mesoporous 2D Covalent Organic Frameworks. J. Am. Chem. Soc. 2007, 129, 12914-12915.

2. Spitler, E. L.; Dichtel, W. R., Lewis acid-catalysed formation of two-dimensional phthalocyanine covalent organic frameworks. Nat. Chem. 2010, 2, 672-677. 
3. Ascherl, L.; Sick, T.; Margraf, J. T.; Lapidus, S. H.; Calik, M.; Hettstedt, C.; Karaghiosoff, K.; Döblinger, M.; Clark, T.; Chapman, K. W.; Auras, F.; Bein, T., Molecular docking sites designed for the generation of highly crystalline covalent organic frameworks. Nat. Chem. 2016, 8, 310-316.

4. Uribe-Romo, F. J.; Hunt, J. R.; Furukawa, H.; Klöck, C.; O’Keeffe, M.; Yaghi, O. M., A Crystalline Imine-Linked 3-D Porous Covalent Organic Framework. J. Am. Chem. Soc. 2009, 131, 4570-4571.

5. $\quad$ Ma, T.; Kapustin, E. A.; Yin, S. X.; Liang, L.; Zhou, Z.; Niu, J.; Li, L.-H.; Wang, Y.; Su, J.; Li, J.; Wang, X.; Wang, W. D.; Wang, W.; Sun, J.; Yaghi, O. M., Single-crystal x-ray diffraction structures of covalent organic frameworks. Science 2018, $361,48-52$.

6. Bessinger, D.; Ascherl, L.; Auras, F.; Bein, T., Spectrally Switchable Photodetection with Near-Infrared-Absorbing Covalent Organic Frameworks. J. Am. Chem. Soc. 2017, 139, 12035-12042.

7. Ascherl, L.; Evans, E. W.; Hennemann, M.; Di Nuzzo, D.; Hufnagel, A. G.; Beetz, M.; Friend, R. H.; Clark, T.; Bein, T.; Auras, F., Solvatochromic covalent organic frameworks. Nat. Commun. 2018, 9, 3802.

8. $\quad$ Feng, X.; Honsho, Y.; Saengsawang, O.; Liu, L.; Wang, L.; Saeki, A.; Irle, S.; Seki, S.; Dong, Y.; Jiang, D., An Ambipolar Conducting Covalent Organic Framework with Self-Sorted and Periodic Electron Donor-Acceptor Ordering. Adv. Mater. 2012, 24, 3026-3031.

9. Dogru, M.; Handloser, M.; Auras, F.; Kunz, T.; Medina, D.; Hartschuh, A.; Knochel, P.; Bein, T., A Photoconductive Thienothiophene-Based Covalent Organic Framework Showing Charge Transfer Towards Included Fullerene. Angew. Chem. Int. Ed. 2013, 52, 2920-2924.

10. Medina, D. D.; Petrus, M. L.; Jumabekov, A. N.; Margraf, J. T.; Weinberger, S.; Rotter, J. M.; Clark, T.; Bein, T., Directional Charge-Carrier Transport in Oriented Benzodithiophene Covalent Organic Framework Thin Films. ACS Nano 2017, 11, 27062713.

11. Spitler, E. L.; Colson, J. W.; Uribe-Romo, F. J.; Woll, A. R.; Giovino, M. R.; Saldivar, A.; Dichtel, W. R., Lattice Expansion of Highly Oriented 2D Phthalocyanine Covalent Organic Framework Films. Angew. Chem. Int. Ed. 2012, 51, $2623-2627$.

12. Zhou, T.-Y.; Xu, S.-Q.; Wen, Q.; Pang, Z.-F.; Zhao, X., One-Step Construction of Two Different Kinds of Pores in a 2D Covalent Organic Framework. J. Am. Chem. Soc. 2014, 136, 15885-15888.

13. Yang, H.; Du, Y.; Wan, S.; Trahan, G. D.; Jina, Y.; Zhang, W., Mesoporous 2D Covalent Organic Frameworks Based on Shape-Persistent Arylene-Ethynylene Macrocycles. Chem. Sci. 2015, 6, 4049-4053.

14. Calik, M.; Sick, T.; Dogru, M.; Döblinger, M.; Datz, S.; Budde, H.; Hartschuh, A.; Auras, F.; Bein, T., From Highly Crystalline to Outer Surface-Functionalized Covalent Organic Frameworks - A Modulation Approach. J. Am. Chem. Soc. 2016, 138, 1234-1239.

15. Bunck, D. N.; Dichtel, W. R., Internal Functionalization of Three-Dimensional Covalent Organic Frameworks. Angew. Chem. Int. Ed. 2012, 51, 1885-1889.

16. Chen, X.; Huang, N.; Gao, J.; Xu, H.; Xu, F.; Jiang, D., Towards covalent organic frameworks with predesignable and aligned open docking sites. Chem. Commun. 2014, 50, 6161-6163.

17. Xu, H.; Gao, J.; Jiang, D., Stable, crystalline, porous, covalent organic frameworks as a platform for chiral organocatalysts. Nat. Chem. 2015, 7, 905-912.

18. Auras, F.; Ascherl, L.; Hakimioun, A. H.; Margraf, J. T.; Hanusch, F. C.; Reuter, S.; Bessinger, D.; Döblinger, M.; Hettstedt, C.; Karaghiosoff, K.; Herbert, S.; Knochel, P.; Clark, T.; Bein, T., Synchronized Offset Stacking: A Concept for Growing LargeDomain and Highly Crystalline 2D Covalent Organic Frameworks. J. Am. Chem. Soc. 2016, 138, 16703-16710.

19. Dalapati, S.; Addicoat, M.; Jin, S.; Sakurai, T.; Gao, J.; Xu, H.; Irle, S.; Seki, S.; Jiang, D., Rational design of crystalline supermicroporous covalent organic frameworks with triangular topologies. Nat. Commun. 2015, 6, 7786.

20. Keller, N.; Bessinger, D.; Reuter, S.; Calik, M.; Ascherl, L.; Hanusch, F. C.; Auras, F.; Bein, T., Oligothiophene-Bridged Conjugated Covalent Organic Frameworks. J. Am. Chem. Soc. 2017, 139, 8194-8199.

21. Bertrand, G. H. V.; Michaelis, V. K.; Ong, T.-C.; Griffin, R. G.; Dinca, M., Thiophene-based covalent organic frameworks. Proc. Natl. Acad. Sci. USA 2013, 110, 4923-4928.

22. Duhović, S.; Dincă, M., Synthesis and Electrical Properties of Covalent Organic Frameworks with Heavy Chalcogens. Chem. Mater. 2015, 27, 5487-5490.

23. Ding, X.; Chen, L.; Honsho, Y.; Feng, X.; Saengsawang, O.; Guo, J.; Saeki, A.; Seki, S.; Irle, S.; Nagase, S.; Parasuk, V.; Jiang, D., An n-Channel Two-Dimensional Covalent Organic Framework. J. Am. Chem. Soc. 2011, 133, $14510-14513$.

24. Cai, S.-L.; Zhang, Y.-B.; Pun, A. B.; He, B.; Yang, J.; Toma, F. M.; Sharp, I. D.; Yaghi, O. M.; Fan, J.; Zheng, S.-R.; Zhang, W.-G.; Liu, Y., Tunable Electrical Conductivity in Oriented Thin Films of Tetrathiafulvalene-Based Covalent Organic Framework. Chem. Sci. 2014, 5, 4693-4700.

25. Gole, B.; Stepanenko, V.; Rager, S.; Grüne, M.; Medina, D. D.; Bein, T.; Würthner, F.; Beuerle, F., Microtubular SelfAssembly of Covalent Organic Frameworks. Angew. Chem. Int. Ed. 2017, 57, 846-850.

26. Wan, S.; Gándara, F.; Asano, A.; Furukawa, H.; Saeki, A.; Dey, S. K.; Liao, L.; Ambrogio, M. W.; Botros, Y. Y.; Duan, X.; Seki, S.; Stoddart, J. F.; Yaghi, O. M., Covalent Organic Frameworks with High Charge Carrier Mobility. Chem. Mater. 2011, 23, 4094-4097.

27. Calik, M.; Auras, F.; Salonen, L. M.; Bader, K.; Grill, I.; Handloser, M.; Medina, D. D.; Dogru, M.; Löbermann, F.; Trauner, D.; Hartschuh, A.; Bein, T., Extraction of Photogenerated Electrons and Holes from a Covalent Organic Framework Integrated Heterojunction. J. Am. Chem. Soc. 2014, 136, 17802-17807.

28. Keller, N.; Calik, M.; Sharapa, D.; Soni, H. R.; Zehetmaier, P. M.; Rager, S.; Auras, F.; Jakowetz, A. C.; Görling, A.; Clark, T.; Bein, T., Enforcing Extended Porphyrin J-Aggregate Stacking in Covalent Organic Frameworks. J. Am. Chem. Soc. 2018, 140, 16544-16552. 
29. Haase, F.; Troschke, E.; Savasci, G.; Banerjee, T.; Duppel, V.; Dörfler, S.; Grundei, M. M. J.; Burow, A. M.; Ochsenfeld, C.; Kaskel, S.; Lotsch, B. V., Topochemical conversion of an imine- into a thiazole-linked covalent organic framework enabling real structure analysis. Nat. Commun. 2018, 9, 2600.

30. Waller, P. J.; AlFaraj, Y. S.; Diercks, C. S.; Jarenwattananon, N. N.; Yaghi, O. M., Conversion of Imine to Oxazole and Thiazole Linkages in Covalent Organic Frameworks. J. Am. Chem. Soc. 2018, 140, 9099-9103.

31. Li, X.; Zhang, C.; Cai, S.; Lei, X.; Altoe, V.; Hong, F.; Urban, J. J.; Ciston, J.; Chan, E. M.; Liu, Y., Facile transformation of imine covalent organic frameworks into ultrastable crystalline porous aromatic frameworks. Nat. Commun. 2018, 9, 2998.

32. Haase, F.; Gottschling, K.; Stegbauer, L.; Germann, L. S.; Gutzler, R.; Duppel, V.; Vyas, V. S.; Kern, K.; Dinnebiera, R. E.; Lotsch, B. V., Tuning the stacking behaviour of a 2D covalent organic framework through non-covalent interactions. Mater. Chem. Front. 2017, 1, 1354-1361.

33. Medina, D. D.; Werner, V.; Auras, F.; Tautz, R.; Dogru, M.; Schuster, J.; Linke, S.; Döblinger, M.; Feldmann, J.; Knochel, P.; Bein, T., Oriented Thin Films of a Benzodithiophene Covalent Organic Framework. ACS Nano 2014, 8, 4042-4052.

34. Jakowetz, A. C.; Hinrichsen, T. F.; Ascherl, L.; Sick, T.; Calik, M.; Auras, F.; Medina, D. D.; Friend, R. H.; Rao, A.; Bein, T., Excited-State Dynamics in Fully Conjugated 2D Covalent Organic Frameworks. J. Am. Chem. Soc. 2019, 141, 11565-11571.

35. Popp, N.; Homburg, T.; Stock, N.; Senker, J., Porous imine-based networks with protonated imine linkages for carbon dioxide separation from mixtures with nitrogen and methane. J. Mater. Chem. A 2015, 3, 18492-18504.

36. Ledbetter, J. W., Infrared Spectra of N-Aryl Imines of $o$-Hydroxybenzaldehyde between 2000 and $1500 \mathrm{~cm}^{-1}$. J. $P$ hys. Chem. 1977, 81, 54-59.

37. Kandambeth, S.; Shinde, D. B.; Panda, M. K.; Lukose, B.; Heine, T.; Banerjee, R., Enhancement of Chemical Stability and Crystallinity in Porphyrin-Containing Covalent Organic Frameworks by Intramolecular Hydrogen Bonds. Angew. Chem. Int. Ed. 2013, 52, 13052-13056.

38. Kütt, A.; Selberg, S.; Kaljurand, I.; Tshepelevitsh, S.; Heering, A.; Darnell, A.; Kaupmees, K.; Piirsalu, M.; Leito, I., pKa values in organic chemistry - Making maximum use of the available data. Tetrahedron Lett. 2018, 59, 3738-3748.

39. Deibert, B. J.; Li, J., A distinct reversible colorimetric and fluorescent low pH response on a water-stable zirconiumporphyrin metal-organic framework. Chem. Commun. 2014, 50, 9636-9639.

40. $\quad$ Chen, L.; He, L.; Ma, F.; Liu, W.; Wang, Y.; Silver, M. A.; Chen, L.; Zhu, L.; Gui, D.; Diwu, J.; Chai, Z.; Wang, S., Covalent Organic Framework Functionalized with 8-Hydroxyquinoline as a Dual-Mode Fluorescent and Colorimetric pH Sensor. ACS Appl. Mater. Interfaces 2018, 10, 15364-15368.

\section{TOC graphics}

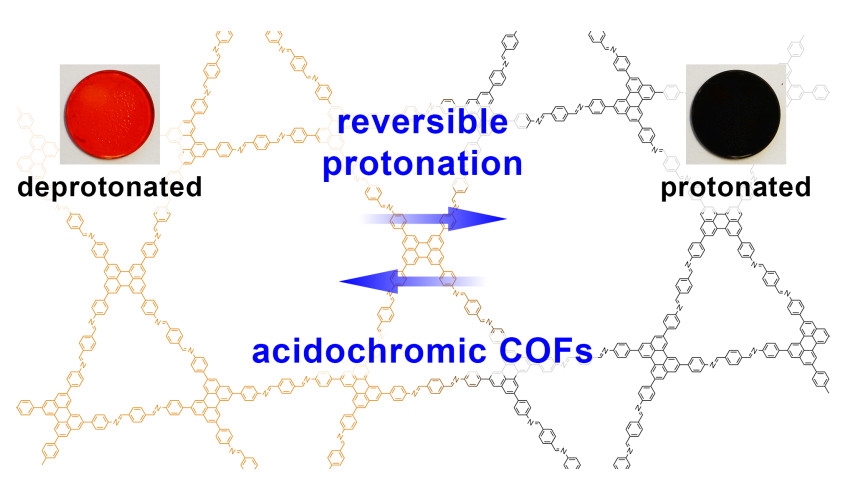

